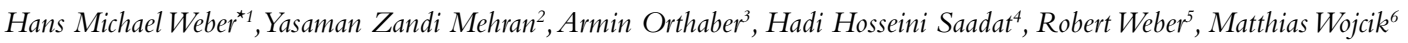

\title{
ANTIVIRALE PHOTODYNAMISCHE THERAPIE BEI COVID-19: EIN NEUER ANSATZ ZUR BEHANDLUNG IN FRÜHEN KRANKHEITSSTADIEN
}

\section{Anti-viral Photodynamic Therapy in Covid-19 Management: A Novel Approach in Treating Patients in Early Infection Stages}

\section{Zusammenfassung}

Aufgrund der weiterhin akuten Covid-19-Pandemie wurde in der hier präsentierten Studie untersucht, ob die Photodynamische Therapie (PDT) mit Riboflavin (Vitamin B2) und einem speziell entwickelten Laser/ LED-Behandlungsset eingesetzt werden kann, um an Covid-19 erkrankte Patienten in frühen Krankheitsstadien therapieren zu können.

Alle 20 Patienten in derVersuchsgruppe zeigten innerhalb der 5 Tage der PDT-Behandlung eine signifikante Verbesserung der klinischen Symptome sowie eine Reduktion derViruslast. 14 von 20 Patienten hatten bereits nach 5 Tagen der Behandlung mit PDT einen negativen QPCR-Test, während die anderen 6 Patienten ebenfalls eine signifikant reduzierte Viruslast aufwiesen. 20 Patienten in der Kontrollgruppe mit konventioneller Versorgung wurden innerhalb von 5 Tagen 3-mal getestet und es konnte keine signifikante Verbesserung festgestellt werden, weder klinisch noch bei der Beurteilung der Viruslast.

Die angewandte Behandlung ist einfach $\mathrm{zu}$ Hause durchführbar und kosteneffektiv. Sie kann zurVorbeugung nach Kontakt mit infizierten Personen oder bei positivem Test, aber auch in frühen Fällen mit leichten bis mittelschweren klinischen Symptomen eingesetzt werden.

\section{Schlüsselwörter}

Covid-19, Photodynamische Therapie (PDT), Riboflavin, Laseruhr, Blau- und Ultraviolettbestrahlung,Viruslast, Prävention

\section{Summary}

Due to the ongoing acute Covid-19 pandemic, the study presented here investigated whether Photodynamic Therapy (PDT) with Riboflavin (vitamin B2) and a specially designed Laser/LED treatment kit can be used to treat patients with Covid-19 at early disease stages.

All 20 patients in the experiment group showed significant improvement in clinical symptoms and viral load assessment within the 5 days of PDT treatment. 14 out of 20 patients had a negative QPCR test after 5 days of treatment with PDT while the other 6 patients also showed significantly reduced viral load. 20 patients in the control group with conventional care were tested 3 times within 5 days and no significant improvement could be seen, neither clinically nor in viral load assessment.

The applied treatment is easy to perform at home and it is cost effective. It can be used for prevention after contact with infected people or in case of positive testing but also in early cases with mild to moderate clinical symptoms.

\section{Keywords}

Covid-19, Photodynamic Therapy (PDT), Riboflavin, laser watch, blue and ultraviolet light irradiation, viral load, prevention 


\section{Was ist Photodynamische Therapie (PDT)?}

Die Photodynamische Therapie (PDT) ist eine vielversprechende Behandlungsmethode im Bereich der Onkologie, Dermatologie und Transfusionsmedizin, deren Effektivität gegen Tumorzellen, aber auch Bakterien, Parasiten und Viren in zahlreichen Studien nachgewiesen werden konnte. Dabei ist die Anwendung von Licht in der Medizin nicht neu: Bereits 1903 wurde der Nobelpreis für die Entdeckung verliehen, dass Ultraviolettlicht erfolgreich zur Behandlung von kutaner Tuberkulose eingesetzt werden kann. Ultraviolettlicht mit niedrigen Wellenlängen (UVB und UVC) wird zudem bereits z.B. zur Sterilisation von Oberflächen eingesetzt, kann aber bekanntermaßen DNA schädigen und Krebs verursachen und deshalb nicht für Anwendungen am Patienten genutzt werden. Aus diesem Grund bedarf es eines etwas komplexeren Vorgehens.

Das Verfahren der Photodynamischen Therapie besteht aus drei Komponenten: Einer durch Licht aktivierbaren Substanz (Photosensitizer), dem im Gewebe vorhandenen Sauerstoff und Licht. Nach Gabe des Photosensitizers (z.B. in Kapselform, per Infusion, in Cremes oder auch Sprühlösungen) wird dieser nach einer gewissen Zeitspanne mit Licht geeigneter Wellenlänge bestrahlt und dadurch aktiviert. Durch photophysikalische Prozesse entstehen in der entsprechenden Körperregion dabei Substanzen - primär reaktive Sauerstoffspezies -, die Viren, Bakterien oder Tumorzellen schädigen und an derVermehrung hindern.

Die Wirkweise wurde insbesondere in der Laborforschung sehr genau belegt. Das Verfahren wird unter anderem in der Transfusionsmedizin eingesetzt, da durch Viren oder Bakterien kontaminierte Blutproben auf diese Weise effizient und kostengünstig sterilisiert werden können.

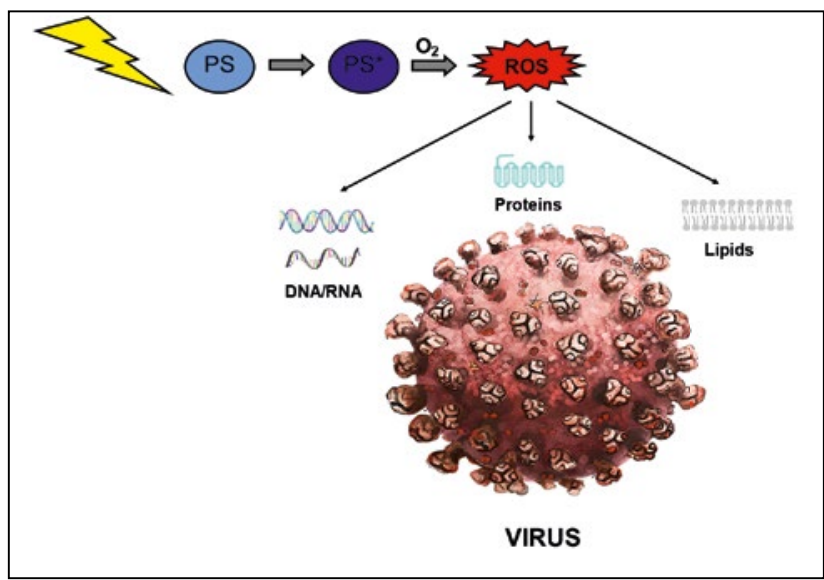

Abb. 1: Antivirale Photodynamische Therapie: Durch die Lichtstimulierung des Photosensitizers (PS) wird dieser aktiviert, wodurch Sauerstoffradikale gebildet werden, die Virusproteine, virale DNA/RNA und Virusmembranen schädigen.
Besonders interessant sind in diesem Zusammenhang zwei Studien aus den USA aus den Jahren 2016 und 2020, in denen nachgewiesen werden konnte, dass auch Coronaviren (MERS-CoV und SARS-CoV-2, dem Erreger der aktuellen Covid-19-Pandemie) unter Laborbedingungen mit äußerst hoher Effizienz durch PDT inaktiviert werden können.

Weitere Vorteile der Photodynamischen Therapie sind, dass sie ohne signifikante Nebenwirkungen angewandt werden kann und Photosensitizer auf natürlich vorkommender und kostengünstiger Basis eingesetzt werden können (Abb. 1).

\section{Riboflavin (Vitamin B2) als Photosensitizer}

Riboflavin, besser bekannt als Vitamin B2, ist ein natürliches Vitamin, das in vielen Nahrungsmitteln enthalten ist und aufgrund einer breiten Studienlage auch in hohen Dosierungen von Behörden wie der US-FDA als sicher eingestuft wird. Bei einer Einzeldosis in Kapselform kann der Körper ca. 25-30 mg absorbieren. Zentral für den medizinischen Einsatz ist eine hohe Bioverfügbarkeit durch die Formulierung als Riboflavin-5-Phosphat (aktive und wasserlösliche Form von Riboflavin).

Riboflavin ist also sicher und nebenwirkungsfrei, kostengünstig und gleichzeitig aber ein sehr potenter Photosensitizer, da die Substanz in Kombination mit ultravioletter und blauer Lichtbestrahlung effektiv gegen Viren und Bakterien wirkt (Abb. 2 u. 3). Diese Wirksamkeit wurde in zahlreichen Studien unter Laborbedingungen nachgewiesen (u.a. bei Coronaviren, s.o.), weshalb die Kombination aus Riboflavin und Lichtbestrahlung in den USA bereits erfolgreich im Bereich der Transfusionsmedizin zur Sterilisation von Blutproben eingesetzt wird.

\section{Gibt es Nebenwirkungen durch die Bestrahlung?}

Die Photodynamische Therapie mit Riboflavin und Bestrahlung mittels UVA- und Blaulicht gilt als sicher und nebenwirkungsfrei. Entscheidend ist in diesem Zusammenhang, dass kein UVB- oder UVC-Licht eingesetzt wird, sondern höhere Wellenlängen, deren Unbedenklichkeit durch diverse Studien belegt ist.

\section{Durchführung der Therapie}

Aufgrund der Daten aus der Laborforschung und Transfusionsmedizin wurde ein Protokoll entwickelt, bei dem Riboflavin-5-Phosphat (100 mg Kapseln, Ultra Botanica/ USA) als Photosensitizer systemisch und lokal (als Sprühlösung) appliziert wird. Anschließend wird das Riboflavin mit 
der sogenannten "Spectra" Laseruhr (W Medical Systems GmbH, Lauenförde) (Abb.4) auf systemischem Wege (über Bestrahlung des Blutes in den Handgelenkarterien) und mittels spezieller Applikatoren für den Nasen-, Mund- und Rachenraum lokal bestrahlt bzw. aktiviert (Abb. 5). Die Applikatoren wurden entsprechend dem Absorptionsspektrum vom Riboflavin mit einer Kombination aus ultravioletten $(375 \mathrm{~nm})$ und blauen $(450 \mathrm{~nm})$ Dioden ausgestattet.

Der Fokus auf die Behandlung der Atemwege als "Eintrittspforte" derViren begründet sich im Fall von Covid-19 von selbst.

Gleichzeitig wird das im Blut zirkulierende Riboflavin systemisch über die Bestrahlung der Handgelenkarterien aktiviert, was insofern von Bedeutung ist, dass Viren auch ins Blut gelangen und innere Organe wie Leber, Herz oder Gehirn angreifen können. In einer Studie von Hagman u. Mitarb. wurde ein Zusammenhang zwischen der Schwere der Erkrankung und der Höhe derVirus-RNA im Blut festgestellt. Die systemische Photodynamische Therapie erfolgt also unter der Prämisse einer Prävention von schweren Verläufen bei Covid-19-Patienten.

Zudem konnte in Studien gezeigt werden, dass die Bestrahlung des Blutes zu einer verbesserten Mikrozirkulation, Immunstimulation, Bildung von Stickstoffmonoxid (NO) und antientzündlichen Effekten führt.

Das hier vorgestellte Behandlungsprotokoll sollte in frühen Krankheitsstadien angewandt werden, um schwere Krankheitsverläufe und intensivmedizinische Behandlung zu vermeiden. Die Behandlung kann leicht vom Patienten selbst im häuslichen Umfeld durchgeführt werden.

\section{Ergebnis der Studie}

In unserer ersten klinischen Studie wurden 20 Patienten im Alter zwischen 18 und 80 Jahren behandelt, die positiv

\section{Anwendungsprotokoll:}

1. Einnahme einer $100 \mathrm{mg}$ Kapsel Riboflavin-5-Phosphat

2. Systemische Behandlung am Handgelenk mit der Spectra Laseruhr (60 min) $1 \mathrm{~h}$ nach Einnahme

3. Herstellung einer Lösung aus einer weiteren Kapsel Riboflavin (100 mg) in einem Glas Wasser (200 ml)

4. Befüllen der Sprühflasche mit der Riboflavinlösung

5. Besprühen der Nasenlöcher (je 3x) mit der Lösung

6. Intensives Spülen und Gurgeln im Mund-Rachen-Raum mit der restlichen Lösung

7. Lösung 15 min einwirken lassen

8. Nase und Mund-Rachen-Raum mit den entsprechenden Applikatoren für jeweils 20 min behandeln

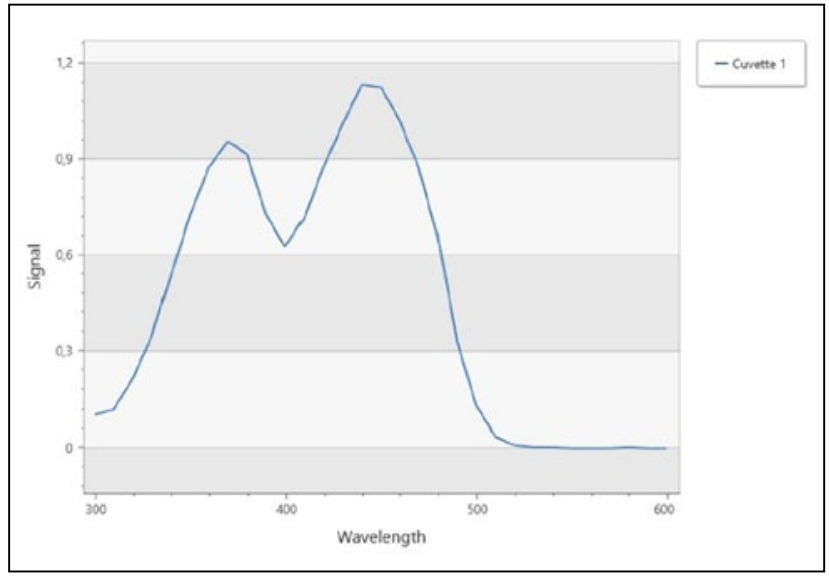

Abb. 2: Lichtabsorption von Riboflavin im UVA- $(375 \mathrm{~nm})$ und Blaubereich $(450 \mathrm{~nm})$

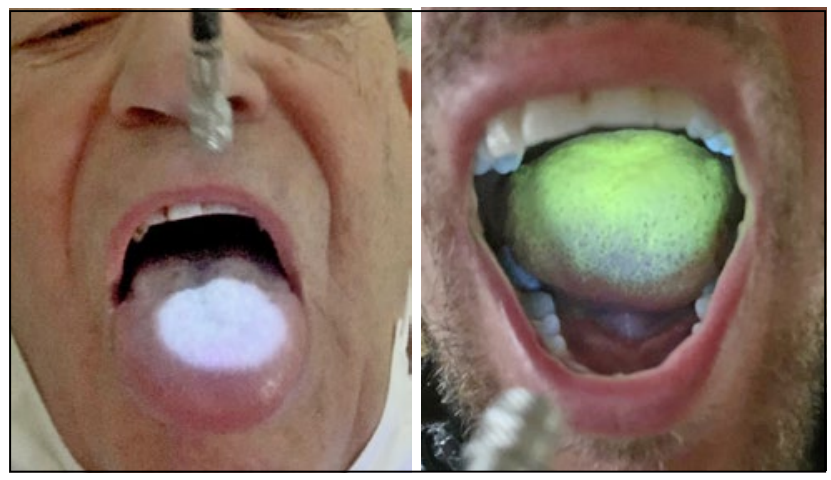

Abb. 3: Bestrahlung der Zunge mit UVA Licht vor (links) und nach (rechts). Besprühen mit einer Riboflavinlösung zeigt eine deutliche Aktivität im rechten Bild.

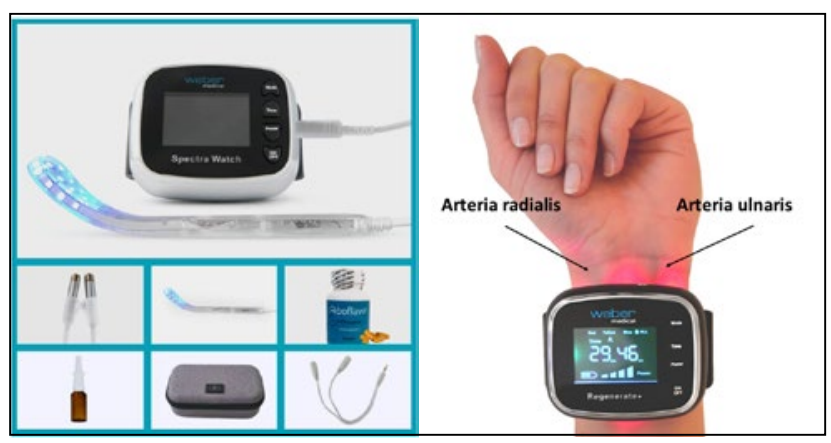

Abb. 4: Behandlungsset und Anwendung der „Laseruhr“

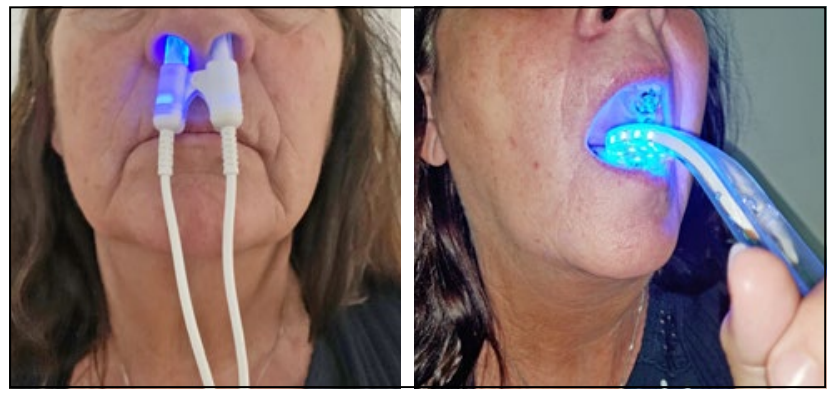

Abb. 5: Behandlung von Nase und Mund-Rachen-Raum 


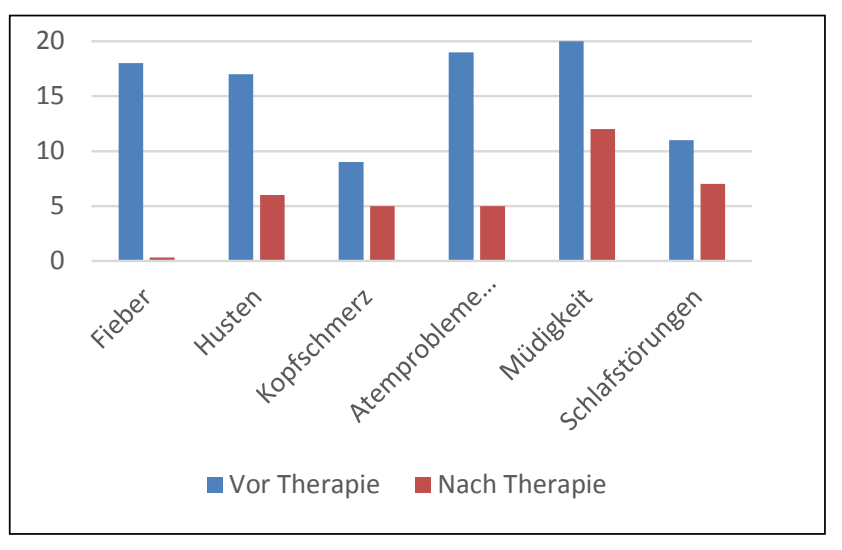

Abb. 6: Klinische Symptome vor und nach PDT

auf Covid-19 getestet wurden und aufgrund stark ausgeprägter klinischer Symptome wie Fieber, Husten, Schmerzen in der Brust, Müdigkeit usw. in ärztlicher Behandlung waren, allerdings noch nicht intubiert oder mit Sauerstoff versorgt werden mussten.

Die PDT-Behandlung wurde einmal täglich über 5 Tage durchgeführt.

Gleichzeitig wurde zumVergleich eine Kontrollgruppe gebildet, in welcher die Patienten die konventionelle Therapie nach den derzeitigen Covid-19-Empfehlungen erhielten.

Zur Verlaufsdiagnostik wurde als Hauptparameter die Viruslast der Patienten herangezogen (Ct-Wert); darüber hinaus wurden die klinischen Symptome dokumentiert.

Abb. 6 zeigt die klinischen Symptome der 20 Patienten, die mit dem PDT-Protokoll behandelt wurden, jeweils vor Beginn und nach Abschluss der Therapie nach 5 Tagen. Bei allen Symptomen lässt sich eine signifikante Besserung erkennen:

Während 18 Patienten zu Beginn über Fieber klagten, hatte keiner der Patienten mehr eine erhöhte Temperatur nach den 5 Behandlungen. 17 Patienten litten zu Beginn unter starkem Husten; nach Therapie noch 6 Patienten. 19 Patienten hatten zu Beginn Atemprobleme und Schmerzen in der Brust; nach der Therapie war diese Symptomatik noch bei 5 Patienten akut. Weniger gravierende Symptome wie Kopfschmerz, Müdigkeit oder Schlafstörungen besserten sich bei ca. der Hälfte der Patienten ebenfalls zügig.

Bei den 20 Patienten in der Kontrollgruppe, die in der gleichen Klinik in Behandlung waren, aber keine zusätzliche PDT erhielten, kam es zu kaum signifikanten Verbesserungen der klinischen Symptome. 2 Patienten mussten auf die Intensivstation verlegt werden; nach 5 Tagen hatten zudem immer noch 10 der restlichen 18 Patienten Fieber. 15 von 18 Patienten litten nach wie vor unter starkem Husten und bei 14 von 18 hielten Atemprobleme und Schmerzen in der Brust weiterhin an.

\section{Viruslastbestimmungen über den Ct-Wert}

Als Maß für die Menge an Virus-RNA kann der beim PCR-Test ermittelte Ct-Wert herangezogen werden. Dieser Wert (Cycle-threshold-Wert) gibt den Vermehrungszyklus der Polymerase-Kettenreaktion an, bei dem zuerst ein exponentieller Anstieg des Fluoreszenz-Signals beobachtet werden kann. Er ist also ein $\mathrm{Ma}$ für die benötigten Schritte zurVervielfältigung des viralen Erbguts. Dabei gilt, je höher der gefundene Ct-Wert ist, desto niedriger ist die ursprünglicheViruskonzentration in der untersuchten Probe. Ct-Werte von $>30$ gelten dabei als Hinweis auf eine niedrige, Werte von $>35$ auf eine sehr niedrige Viruskonzentration. Die ursprüngliche Virenmenge wiederum beeinflusst, wie ansteckend eine infizierte Person ist. Eine hohe Viruskonzentration im Nasen-Rachen-Raum sorgt im Normalfall auch für eine hohe Infektiosität.

\section{Ergebnisse der Viruslastmessungen}

Tab. 1 zeigt die Ergebnisse derViruslastbestimmungen bzw. die Ct-Werte der 20 Patienten, die mit dem PDT-Protokoll behandelt wurden. Der Ct-Wert stieg bei allen $20 \mathrm{~Pa}-$ tienten im Laufe der 5 Tage sukzessive signifikant an. Im Durchschnitt stieg der Ct-Wert zwischen Tag 1 und 3 um 3,99 Punkte und zwischen Tag 3 und 5 um 4,98 Punkte. Nach 5 Tagen hatten 14 der 20 Patienten bereits ein negatives Testergebnis, während die restlichen 6 Patienten nur noch eine niedrige Viruslast hatten.

In der Kontrollgruppe konnte dagegen keine signifikante Reduktion derViruslast festgestellt werden (Anstieg des Ct-Werts im Durchschnitt um 0,37 bzw. 0,48 Punkte). Nach den 5 Tagen hatten alle Patienten der Kontrollgruppe weiterhin ein positives Testergebnis.

\section{Fazit}

Nachdem bereits in zahlreichen In-vitro-Studien die Effektivität von PhotodynamischerTherapie gegen verschiedeneViren einschließlich des neuen Coronavirus (SARSCoV-2) nachgewiesen werden konnte, ist die hier präsentierte Studie der erste Beleg für die Wirksamkeit dieses Therapieansatzes bei akut infizierten Patienten: Bei allen mit PDT behandelten Patienten konnte dieViruslast binnen weniger Tage signifikant gesenkt werden, bei 70\% der Patienten sogar soweit, dass nach 5 Tagen bereits ein negatives Testergebnis vorlag. Gleichzeitig konnte bei allen 20 Patienten ein starker Rückgang der klinischen Symptome beobachtet werden. 
Tab. 1: QPCR Test / Ct Wert an Tag 1-5

\begin{tabular}{|l|c|c|c|c|c|c|c|}
\hline Alter & Geschlecht & Tag 0 & Tag 1 & Tag 2 & Tag 3 & Tag 4 & Tag 5 \\
\hline 49 & F & + & 37.2 & 38.9 & Negativ & Negativ & Negativ \\
\hline 52 & M & + & 35.2 & 34.5 & 36.8 & 37.8 & Negativ \\
\hline 24 & F & + & 28.3 & 30.3 & 35.7 & 35.1 & Negativ \\
\hline 18 & F & + & 35.1 & 39.3 & Negativ & Negativ & Negativ \\
\hline 37 & F & + & 28.3 & 30.5 & 32.2 & 35.2 & Negativ \\
\hline 27 & F & + & 29.8 & 30.2 & 31.2 & 33.4 & Negativ \\
\hline 40 & M & + & 29.7 & 29.2 & 35.4 & 37.2 & Negativ \\
\hline 57 & F & + & 29.2 & 29.2 & 34.3 & 37.8 & Negativ \\
\hline 50 & M & + & 22.7 & 27.3 & 25.6 & 30.1 & 33.7 \\
\hline 31 & M & + & 27.1 & 30.2 & 32.2 & 36.7 & Negativ \\
\hline 53 & M & + & 23.9 & 24.6 & 27.7 & 34.4 & 35.1 \\
\hline 80 & F & + & 34.6 & Negativ & Negativ & 38.8 & 39.1 \\
\hline 39 & M & + & 34.1 & 35.7 & 38.8 & 39.1 & Negativ \\
\hline 25 & F & + & 24.1 & 26.6 & 29.7 & 35.7 & Negativ \\
\hline 50 & F & + & 26.7 & 28.8 & 33.4 & 37.2 & Negativ \\
\hline 67 & F & + & 35.5 & 34.3 & 36.7 & 38.1 & Negativ \\
\hline 39 & F & + & 35.4 & 37.1 & 38.9 & 39.2 & Negativ \\
\hline 45 & F & + & 31.2 & 32.9 & 35.4 & 35.5 & 37.2 \\
\hline 39 & M & + & 21.4 & 25.2 & 23.4 & 26.9 & 32.2 \\
\hline 37 & F & + & 23.6 & 27.9 & 25.4 & 26.2 & 29.7 \\
\hline
\end{tabular}

Im Vergleich dazu gab es bei einer Kontrollgruppe mit 20 Patienten, die eine konventionelle medikamentöse Therapie erhielten, innerhalb von 5 Tagen keinen signifikanten Rückgang der Viruslast oder der klinischen Symptome, während 2 Patienten sogar intensivmedizinisch betreut werden mussten.

Die Behandlung ist praktisch nebenwirkungsfrei, kostengünstig und kann vom Patienten selbst angewendet werden. Neben der Behandlung einer akuten Infektion kann das hier vorgestellte Protokoll auch zur Vorsorge eingesetzt werden, z.B. nach Kontakt mit potenziell infizierten Personen.

Ein weiterer Vorteil der Photodynamischen Therapie besteht darin, dass der gleiche Ansatz voraussichtlich auch bei anderen Erkältungs- und Grippeviren oder Bakterien wirksam sein sollte, was sich bereits in ersten Patiententests zu bestätigen scheint.

Neben einer präventiven Impfstrategie kann die PDT folglich ein wichtiger Baustein sein, um akut infizierte Patienten rasch und effektiv behandeln zu können und somit soziale und wirtschaftliche Kosten zu reduzieren; insbesondere, da weiterhin kaum effektive Therapieansätze oder Medikamente für akut Infizierte zur Verfügung stehen. Darüber hinaus kann die PDT potenziell auch zu einem natürlichen Immunisierungseffekt führen; Untersuchungen dazu sowie weitere Studien mit einer größeren Patientenanzahl wurden bereits initiiert.

Die Kombination aus präventiven Maßnahmen wie Impfungen und Abstandsregeln sowie therapeutischen Ansätzen wie der PDT für akut infizierte Patienten ist nach der Ansicht der Studienautoren der Schlüssel zur erfolgreichen Bekämpfung der Covid-19-Pandemie.

Die PDT Behandlung sollte dabei in frühen Krankheitsstadien eingesetzt werden, um die Entwicklung von schweren Krankheitsverläufen mit Krankenhausaufenthalt vorzubeugen, sodass Patienten ihre Erkrankung in der häuslichen Umgebung auskurieren können, ohne das Gesundheitssystem noch stärker zu belasten.

\section{Die Originalstudie finden Sie hier:}

https://medclinres.org/pdfs/2020/successful-reductionof-sars-cov-2-viral-load-by-photodynamic-therapy-pdtverified-by-qpcr-a-novel-approach-in-treating-patientsin-early-infection-stages-mcr-20.pdf 


\section{Literatur}

1. Allison RR, Sibata $\mathrm{CH}$ : Oncologic photodynamic therapy photosensitizers: A clinical review. Photodiagnosis Photodyn Ther 2010; 7: 61-75.

2. Kharkwal GB, Sharma SK, Huang YY et al.: Photodynamic Therapy for Infections: Clinical Applications. Lasers Surg Med 2011; 43: 755-767.

3. Allison RR, Downie GH, Cuenca R et al.: Photosensitizers in clinical PDT. Photodiagnosis Photodyn Ther 2004; 1:27-42.

4. Suwannasom N, Kao I, Pruss A et al.: Riboflavin: The Health benefits of a forgotten natural Vitamin. Int J Mol Sci 2020; 950: 1-22.

5. Hu X, HuangYY,WangY et al.:Antimicrobial Photodynamic Therapy to Control Clinically Relevant Biofilm. Infections. Front Microbiol 2018; 9: 1299.

6. Wiehe A, O’Brien JM, Senge MO:Trends and targets in antiviral phototherapy. Photoche. Photobiol Sci 2019; 18: 25652612.

7. Unna K, Greslin JG: Studies on the toxicity and pharmacology of riboflavin. J Pharmacol Exp Ther 1942; 76: 75-78.

8. Zempleni J, Galloway JR, McCormick DB: Pharmacokinetics of orally and intravenously administered riboflavin in healthy humans. Am J Clin Nutr 1996; 63: 54-66.

9. Boehnke C, Reuter U, Flach U et al.: High-dose riboflavin treatment is efficious in migraine prophylaxis: an open study in a tertiary care centre. Eur J Neurol 2004; 11: 475-477.

10. Powers HJ: Riboflavin (vitamin B-2) and health. Am J Clin Nutr 2003; 77: 1352-1360.

11. Wainwright M: Photoinactivation of viruses. Photochem Photobiol Sci 2004; 3: 406-411.

12. Ben-Hur E, RP Goodrich: Pathogen Reduction in Blood for Transfusion Using Photodynamic Treatments. In: Hamblin MR, Jori G, eds.: Photodynamic Inactivation of Microbial Pathogens. Medical and Environmental Applications, Cambridge, UK: RSC Publishing; 2011: 235-263.

13. Schlenke P: Pathogen Inactivation Technologies for Cellular Blood Components: An Update. Transfus Med Hemother 2014; 41: 309-325.

14. Keil SD, Bowen R, Marschner S: MERS-CoV in plasma products using a riboflavin-based and ultraviolet light-based photochemical treatment. Transfusion 2016; 56: 2948-2952.
15. Schuyler R: Use of riboflavin for photoinactivation of pathogens in blood components. Transfus Apher Sci 2001; 25: 189190.

16. Marschner S, Goodrich R: Pathogen Reduction Technology Treatment of Platelets, Plasma and Whole Blood Using Riboflavin and UV Light. Transfus Med Hemother 2011; 38 8-18.

17. Keil SD, Ragan I, Yonemura S et al.: Inactivation of severe acute respiratory syndrome coronavirus 2 in plasma and platelet products using a riboflavin and ultraviolet light-based photochemical treatment.Vox Sang 2020; 6: 495-501.

18. Rezaie A, Leite GGS, Melmed GY et al.: Ultraviolet A light effectively reduces bacteria and viruses including coronavirus. PLoS One 2020; 15: 1-11.

19. Mittermayr R, Osipov A, Piskernik C et al.: Blue Laser Light Increases Perfusion of a Skin Flap Via Release of Nitric Oxide from Hemoglobin. Mol Med 2007; 13: 22-29.

20. Loscalzo J: The antithrombotic effect of nitric oxide. In: Catravas JD, Callow AD, Ryan US, eds.: Vascular Endothelium. NATO ASI Series (Series A: Life Sciences). Vol 294. Berlin: Springer; 1998; 294: 99-102.

21. Ahmad I Q, Fasihullah Q,Vaid FHM: Effect of light intensity and wavelengths on photo degradation reactions of riboflavin in aqueous solution. J Photochem Photobiol B 2006; 82: 21-27.

22. Litscher G, Litscher D:A Laser Watch for Simultaneous Laser Blood Irradiation and Laser Acupuncture at the Wrist. Integr Med Int 2016; 3: 75-81.

23. Mikhaylov VA:The use of Intravenous Laser Blood Irradiation (ILBI) at 630-640 $\mathrm{nm}$ to prevent vascular diseases and to increase life expectancy. Laser Ther 2015; 24: 15-26.

24. Amjadi A, Mirmiranpour $\mathrm{H}$, Sobhani SO et al.: Intravenous laser wavelength radiation effect on LCAT, PON1, catalase, and FRAP in diabetic rats. Lasers Med Sci 2020;35: 131-138.

25. Hagman K, Hedenstierna M, Gille-Johnson P et al.: Severe Acute Respiratory Syndrome Coronavirus 2 RNA in Serum as Predictor of Severe Outcome in Coronavirus Disease 2019: A Retrospective Cohort Study Clin Infect Dis 2020: ciaa1285

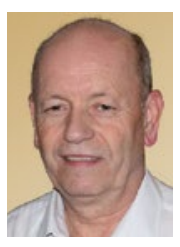

Dr. med. Dipl. Chem. Michael Weber

President for Medical and Clinical Applications der International Society for Medical Laser Applications

(ISLAtranscontinental)

Sohnreystrasse 4, 37697 Lauenförde

Internet: www.isla-laser.org 